Provided for non-commercial research and education use. Not for reproduction, distribution or commercial use.

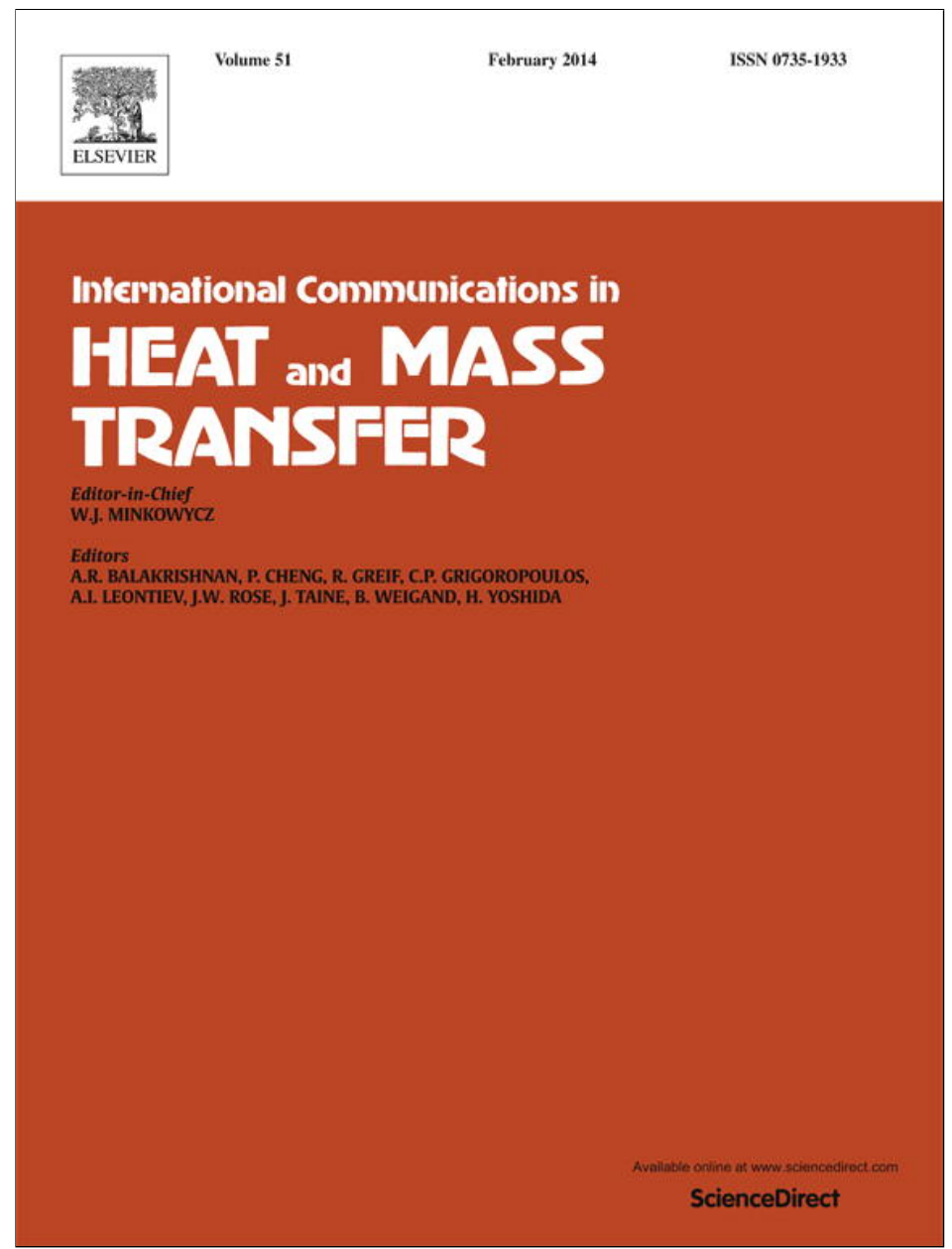

This article appeared in a journal published by Elsevier. The attached copy is furnished to the author for internal non-commercial research and education use, including for instruction at the authors institution and sharing with colleagues.

Other uses, including reproduction and distribution, or selling or licensing copies, or posting to personal, institutional or third party websites are prohibited.

In most cases authors are permitted to post their version of the article (e.g. in Word or Tex form) to their personal website or institutional repository. Authors requiring further information regarding Elsevier's archiving and manuscript policies are encouraged to visit:

http://www.elsevier.com/authorsrights 


\title{
An evaporation model for oscillating spheroidal drops $s^{\text {is }}$
}

\author{
S. Tonini, G.E. Cossali* \\ Engineering Department, University of Bergamo, Viale Marconi 5, 24044 Dalmine, Italy
}

\section{A R T I C L E I N F O}

Available online 19 December 2013

\section{Keywords:}

Spheroidal drop

Evaporation

Oscillating drop

\begin{abstract}
A B S T R A C T
The evaporation process of a liquid spheroidal drop floating in a gaseous atmosphere has been modelled, accounting for the oscillation between oblate and prolate states. A previously developed exact solution for the heat and mass transfer equations has been extended to investigate the effect of oscillation on drop evaporation under the assumption of quasi steady-state conditions and the results are compared with approximate models from the open literature. The validity of the quasi steady-state assumption is discussed, deriving, for different fluids, the range of drop temperature and size and gas temperature where it is reasonably acceptable.
\end{abstract}

(c) 2013 Elsevier Ltd. All rights reserved.

\section{Introduction}

The growing practical interest in spray evaporation and combustion has motivated the request of a detailed understanding of the different phenomena taking place when a liquid droplet floats in a gaseous atmosphere, with an associated increasing demand for analytical/empirical correlations describing the interphase transfer of mass, momentum and energy [1].

The process of liquid drop vaporization has attracted the researchers since the nineteenth century. The simplest model for the droplet evaporation rate was proposed by Maxwell back in 1877 [2], which suggested that the driving force for liquid evaporation is the difference in vapour concentration between the drop surface and the free stream and the process is exclusively controlled by the diffusion mechanism.

Since then, a variety of different models have been proposed in order to capture the complexity of the physical phenomena involved in the process, including the bulk motion of the gas surrounding the droplet (Stefan flow) [3], the heat and mass diffusion in the droplet interior [4], the liquid composition [5,6], gas stream effect [7] and highpressure effect [8,9]. Refer to [10] for a recent review of the main developments in modelling droplet heating and evaporation.

One assumption that yet prevails in most of the theoretical/empirical models widely used in commercial CFD codes for dispersed phase (like sprays, or particle laden flows) application is that liquid droplets maintain spherical shape while interacting with the gaseous phase [7]. However, significant shape deformations are expected and observed while liquid drops interact with the carrier phase, and these deformations are of fundamental importance for understanding many natural and industrial processes involving spray droplets [11,12].

Non-spherical liquid drops are unstable and the opposing effects of surface tension and inertia cause periodic or non-periodic variation of the drop shape, which is referred to as drop oscillation and it is found

\footnotetext{
is Communicated by W.J. Minkowycz.

* Corresponding author.

E-mail address: cossali@unibg.it (G.E. Cossali).
}

to strongly influence heat, momentum and mass transfer between liquid drops and the surrounding gas [13]. Oscillation can become important in atomisation systems where the liquid is first disintegrated into small ligaments, which then oscillate towards the asymptotic attainment of an equilibrium spherical shape [14].

One of the pioneering works on non-spherical droplet dynamics was that of Lamb [15], which led to an expression for the natural frequency of infinitesimal amplitude oscillations of an inviscid drop immersed in an inviscid quiescent environment.

Afterwards, a considerable amount of work has been done on the dynamics of oscillating drops; refer to [16-22] for reviews on numerical and experimental contributions to this field. Effect of viscosity was considered by many researchers, among other results it was shown that viscosity effects are responsible of a relatively quick damping of the highest oscillation modes, then living only the oblate-prolate mode $(n=2)$ to survive [23].

All these works address the issue of oscillating drop/ligament dynamics under non-evaporating conditions. When the drops are exposed to hot gas, the heat transfer and the consequent evaporation could affect and be affected by oscillations [24-26].

Despite the large amount of work done over the last decades on nonevaporating liquid drop oscillation, to the best of authors' knowledge only few papers can be found in the open scientific literature addressing the effect of evaporation on oscillating drops [27-29], and the few available experimental data-sets do not report all the necessary information for model comparison.

Deng et al. [30] proposed one of the early numerical studies on this subject with a two-dimensional numerical model investigating the dynamics of non-evaporating and evaporating liquid ligaments undergoing deformation/breakup and oscillations under viscous convective flows. The results showed that the dynamics of ligament deformation was basically unaffected by vaporization, however the evaporation rates (per unit area) are greater for deformed drops.

Mashayek [31] suggested a correlation for the rate of evaporation of deformed drop based on the results from numerical simulations, which 


\section{Nomenclature}

Greek symbols

$\begin{array}{lll}\alpha & \text { thermal diffusivity } & \mathrm{m}^{2} / \mathrm{s} \\ \beta & \text { surface area ratio } & - \\ \gamma & \text { specie flux } & \mathrm{kg} / \mathrm{m}^{2} \mathrm{~s} \\ \epsilon_{n} & \text { non-dimensional disturbance amplitude } & - \\ \varepsilon & \text { deformation parameter } \varepsilon=\frac{a_{z}}{a_{r}} & - \\ \zeta, \theta, \varphi & \text { spheroid coordinates } & - \\ \rho & \text { density } & \mathrm{kg} / \mathrm{m}^{3} \\ \sigma & \text { surface tension } & \mathrm{kg} / \mathrm{s}^{2} \\ \tau & \text { oscillation period } & \mathrm{S} \\ \chi & \text { mass fraction } & - \\ \omega & \text { oscillation frequency } & 1 / \mathrm{s} \\ \Gamma & \text { evaporation enhancement } & - \\ \Delta & \text { relative difference } & - \\ \Theta & \text { non-dimensional evaporation rate parameter }- \\ \Xi & \text { mean curvature } & - \\ \Pi & \text { non-dimensional vapour flux } & -\end{array}$

\section{Roman symbols}

$\begin{array}{lll}a & \text { perturbation parameter } & - \\ a_{r}, a_{z} & \text { spheroid axes } & \mathrm{m} \\ C_{p} & \text { specific heat } & \mathrm{J} / \mathrm{kgK} \\ D_{v} & \text { diffusivity } & \mathrm{m}^{2} / \mathrm{s} \\ k & \text { thermal conductivity } & \mathrm{W} / \mathrm{mK} \\ \dot{m}_{e v} & \text { evaporation rate } & \mathrm{kg} / \mathrm{s} \\ n & \text { oscillation mode } & - \\ P_{n} & \text { Legendre polynomials } & - \\ R & \text { drop radius } & \mathrm{m} \\ t & \text { time } & \mathrm{s} \\ T & \text { temperature } & \mathrm{K} \\ U & \text { velocity } & \mathrm{m} / \mathrm{s} \\ \chi, y, z & \text { Cartesian coordinates } & -\end{array}$

$\begin{array}{ll}\text { Subscripts } \\ \alpha & \text { species index } \\ \text { conv } & \text { convective } \\ \text { diff } & \text { diffusive } \\ c d & \text { convective-diffusive } \\ \text { evap } & \text { evaporation } \\ g & \text { gaseous } \\ l & \text { liquid } \\ \text { oscil } & \text { oscillation } \\ 0, s & \text { surface } \\ v & \text { vapour } \\ \infty & \text { infinity }\end{array}$

$$
\begin{array}{ll}
\text { Superscripts } \\
\sim & \text { non-dimensional } \\
H & \text { heat transfer }
\end{array}
$$

showed that the mass flux varies along the surface of the deformed drop. The Author proposed a correlation to express the mass flux as a function of the surface curvature, based on the suggestion of Lian and Reitz [32] who studied the instability of evaporating liquid jets, postulating that the deformed surface may be locally considered as the surface of a spherical drop having the same mean curvature as that of the deformed surface, and that the local flux would be that of a spherical drop with that curvature radius.
Recently [33], it has been shown that an analytical solution of the steady state heat and mass transfer equations exists for spheroidal (oblate and prolate) drops floating in a gaseous atmosphere, and the local evaporation rate was exactly correlated with the local surface curvature.

The evaporation from free oscillating particles was investigated in [34], showing that the increase in the evaporation rate of an oscillating drop is proportional to the square of the surface disturbance amplitude and is larger for higher oscillation modes, and that the period of oscillation is decreased by evaporation, while the dominant mode of oscillation remains the same as that for a non-evaporating drop.

The present work was motivated by the necessity to include the above described complex drop evaporation mechanisms in spray numerical simulations, using relatively simple sub-models for predicting the inter-phase phenomena taking place during the spray evolution. The following sections report the mathematical model, the derivation of analytical expressions for the instantaneous and average evaporation rate and heat rate from oscillating spheroidal liquid drops, the comparison against the predictions of previously available models and the derivation of conditions for model applicability for different fluids. The main conclusions are then briefly summarised.

\section{The instantaneous vapour flux and heat rate for spheroidal drops}

For a liquid drop made of a single component floating in a gaseous atmosphere, the species conservation equations can be written [35]:

$\rho U_{j} \nabla_{j} \chi_{\alpha}=\nabla_{j}\left(\rho D_{v} \nabla_{j} \chi_{\alpha}\right)$

where $\alpha=v$, g refers to the vapour and gaseous phases respectively, while $\chi_{\alpha}=\frac{\rho_{\alpha}}{\rho}$ is the mass fraction and $D_{v}$ is the binary diffusion coefficient; the symmetry of the diffusion coefficients $D_{v}=D_{v g}=D_{g v}$ for a binary mixture is imposed according to [35].

In the following, gas density is assumed to be constant, according to the majority of evaporation models for spray simulations [36]. Accounting for density gradient effect on spherical droplet evaporation [37] led to the conclusion that the constant gas density assumption may become questionable for very high gas temperature evaporating conditions.

Setting to constant values the vapour mass fraction at drop surface $\left(\chi_{v}=\chi_{v, s}\right)$ and at infinite distance from the drop $\left(\chi_{v}=\chi_{v, \infty}\right)$, an analytical solution of (1) was proposed in [33], through the use of prolate and oblate spheroidal coordinate systems, defined as:

$x=a A(\xi) \sin (\theta) \cos (\varphi)$

$y=a A(\xi) \sin (\theta) \sin (\varphi)$

$z=a B(\xi) \cos (\theta)$

where:

$A(\xi)=\cosh (\xi) ; B(\xi)=\sinh (\xi) ; \quad$ for oblate case $A(\xi)=\sinh (\xi) ; B(\xi)=\cosh (\xi) ;$ for prolate case

In these coordinate systems, the spheroid surface equation is $\xi=\xi_{0}$, and the above-mentioned B.C. are:

$\chi_{v}\left(\xi_{0}, \theta, \varphi\right)=\chi_{v, s} ; \quad \chi_{v}(\infty, \theta, \varphi)=\chi_{v, \infty}$.

The steady state analytical solution of the balance Eq. (1) provides the following form for the local instantaneous vapour flux:

$\gamma_{v, s}=\frac{\rho D_{v}}{R_{0}} \frac{\varepsilon^{2 / 3}}{\left[1-\left(1-\varepsilon^{2}\right) \sin ^{2}(\theta)\right]^{1 / 2}} \Gamma(\varepsilon) \ln \frac{1-\chi_{v, \infty}}{1-\chi_{v, s}}$

where the deformation parameter $\varepsilon$ is defined as:

$\varepsilon=\frac{a_{z}}{a_{r}}$ 
and $a_{z}$ and $a_{r}$ are respectively the axial and radial spheroid axes, see Fig. 1 for reference, and the parameter $\Gamma(\varepsilon)$ is defined as follows:

$$
\Gamma(\varepsilon)=\begin{array}{cc}
\frac{1}{\sqrt{\left(1-\varepsilon^{2}\right)}} & \varepsilon=1 \text {, Sphere } \\
\frac{\sqrt{\left(\varepsilon^{2}-1\right)}}{\varepsilon^{1 / 3}\left(\pi-2 \arctan \left(\sqrt{\left(\frac{1+\varepsilon}{1-\varepsilon}\right)}\right)\right.} & \varepsilon<1 \text {, Oblate } \\
\frac{\varepsilon^{1 / 3}\left[\ln \left(\sqrt{\left(\frac{\varepsilon+1}{\varepsilon-1}\right)}+1\right)-\ln \left(\sqrt{\left(\frac{\varepsilon+1}{\varepsilon-1}\right)}-1\right)\right]}{\varepsilon>1 \text {, Prolate }} &
\end{array}
$$

Integration of the vapour flux over the entire drop surface yields the value of the evaporation rate, that in non-dimensional form is:

$\hat{m}_{e v}=\frac{\dot{m}_{e v}}{4 \pi R_{0} D_{v} \rho}=\Gamma(\varepsilon) \ln \left(\frac{1-\chi_{v, \infty}}{1-\chi_{v, s}}\right)$.

To notice that the non-dimensional evaporation rate for a spherical droplet is simply:

$\hat{m}_{e v}^{\text {sphere }}=\ln \left(\frac{1-\chi_{v, \infty}}{1-\chi_{v, s}}\right)$.

In an analogous way [33] the non-dimensional sensible heat rate assumes the following form:

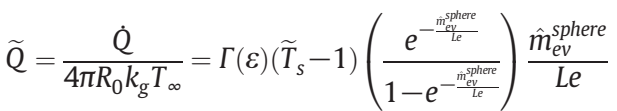

being Le the Lewis number, $L e=\frac{\alpha}{D_{v}}$ and $T^{\mathrm{s}}=T_{\mathrm{s}} / T_{\infty}$.

From Eqs. (3) and (4), it can be seen that the evaporation rate of a prolate spheroid is always larger than that of an oblate spheroid having both the same volume and the same surface (see [33]).

An approximate model for evaporation from a deformed drop was proposed by Mashayek in [31] and it was used to estimate the evaporation rate from oscillating droplets. Following a method suggested by Lian and Reitz [32], a correlation was proposed to express the instantaneous local mass flux as a function of the local mean curvature of the surface:

$\gamma_{v, s}^{\text {Mashayek }[31]}=\Xi \frac{k_{g}}{2 R_{0} C_{p g}} \hat{m}_{e v}^{\text {sphere }}$ where $\Xi$ is the mean curvature [38], which for a spheroid is equal to:

$\Xi=\varepsilon^{4 / 3} \frac{\left(\varepsilon^{2}-1\right) \sin ^{2}(\theta)+2}{\left[\left(\varepsilon^{2}-1\right) \sin ^{2}(\theta)+1\right]^{3 / 2}}$.

Fig. 2 shows the comparison between the exact solution for spheroidal drops, Eq. (2), and the approximate model [31] in terms of the nondimensional local vapour flux along the drop surface, defined as:

$\Pi=\frac{\gamma_{v, s}}{\frac{k_{g}}{2 R_{0} C_{p g}} \hat{m}_{e v}^{\text {sphere }}}$.

To be noticed that for the approximate model [31], $\Pi$ reduces to the value of the mean curvature $\Xi$. Three values of the surface ratio $\beta$, defined as:

$\beta=\frac{A_{\text {spheroid }}}{A_{\text {sphere }}}=\frac{1}{2 \varepsilon^{2 / 3}}\left\{\begin{array}{cc}1+\frac{\varepsilon^{2} \ln \left(\frac{1+\sqrt{1-\varepsilon^{2}}}{\varepsilon}\right)}{\sqrt{1-\varepsilon^{2}}} & \text { Oblate } \\ 1+\frac{\varepsilon^{2} \arctan \left(\sqrt{\varepsilon^{2}-1}\right)}{\sqrt{\varepsilon^{2}-1}} & \text { Prolate }\end{array}\right.$

are selected equal to $1.05,1.1$ and 1.2, which correspond to different values of the deformation parameter $\varepsilon$ for the corresponding isosurface oblate and prolate drops. The graphs are presented as function of the azimuthal coordinate $\theta$ between $-\pi / 2$ and $\pi / 2$. The results enlighten that the approximate model predicts larger variations of the vapour flux along the surface, compared to the exact solution.

However the non-dimensional evaporation rate, obtained by the vapour flux integration along the drop surface, calculated by the approximate model differs from the exact value by less than $15 \%$, as reported in the figure by the non-dimensional parameter $\Theta=\frac{m_{e v}}{\frac{m_{e v e r e}}{\text { selper }}}$

\section{The evaporation model for oscillating drops}

The frequency of the oscillation modes of a liquid drop in gaseous environment can be described (for small oscillation amplitude) by the generalised Lamb equation [15].

$\omega_{n}^{2}=\frac{n(n+1)(n-1)(n+2)}{(n+1) \rho_{l}+n \rho_{g}} \frac{\sigma}{R_{0}^{3}}$

where $\rho_{l}$ and $\rho_{g}$ refer to the densities of the liquid and gas fluids respectively, and the index $n$ refers to the oscillation modes: $n=0$ stands for a pure expansion (only for bubbles), $n=1$ refers to translation mode,
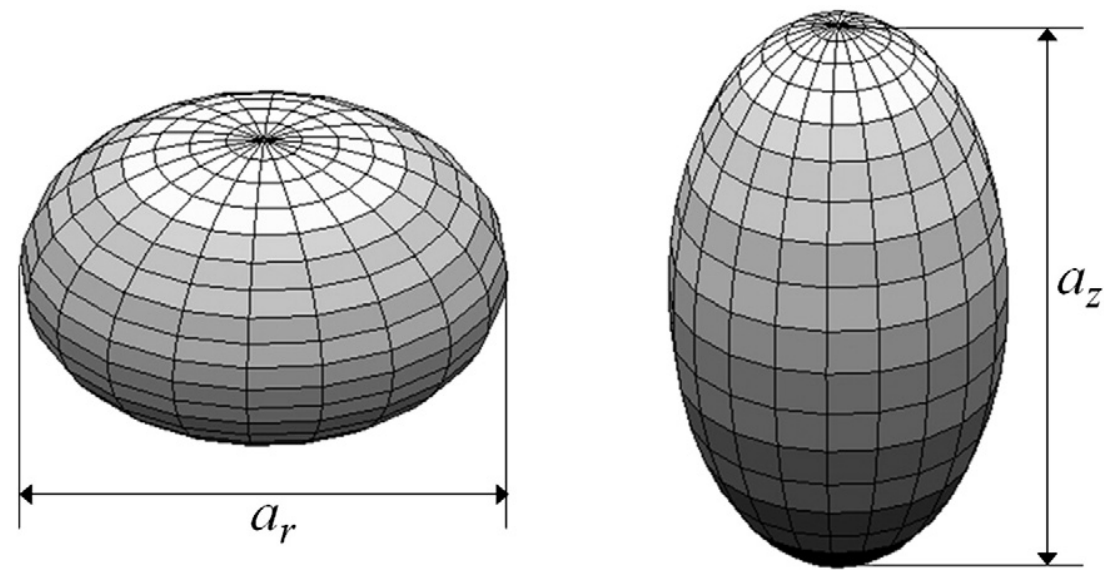

Fig. 1. Oblate (left) and prolate (right) spheroids and definition of axial $\left(a_{z}\right)$ and radial $\left(a_{r}\right)$ spheroid semi-axes. 


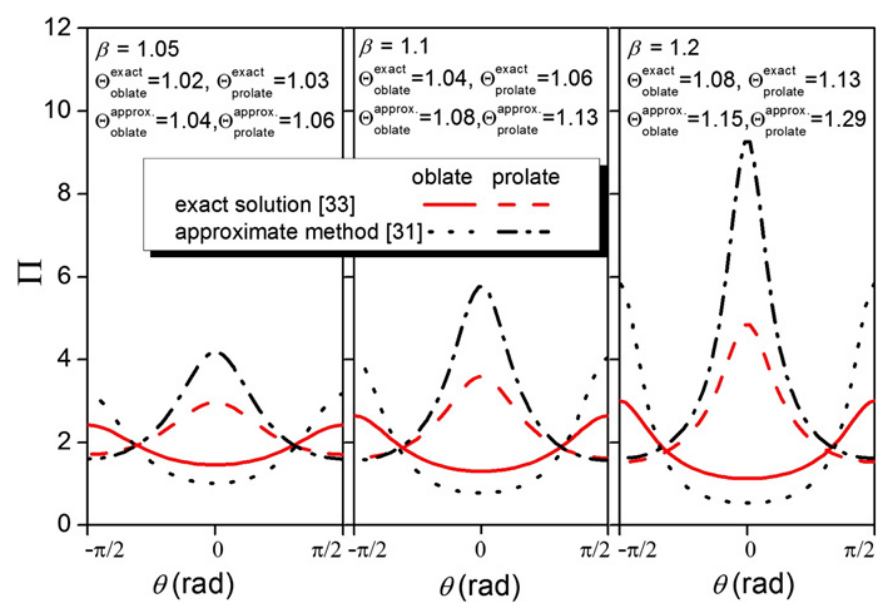

Fig. 2. Non-dimensional vapour flux along the drop surface, predicted by the exact solution [33] and the approximate method of Mashayek [31] as function of the surface ratio $\beta$, for both oblate and prolate drops.

without a corresponding frequency, $n=2$ refers to oblate-prolate mode, and finally $n>2$ describes more complex modes.

Considering the effect of liquid viscosity, according to [16], the higher modes, for liquid drops oscillating in a gaseous environment, are quickly damped and the mode $n=2$ is the only long lasting one. Under the conditions that $\rho_{l} \gg \rho_{g}$ Eq. (10) for the mode $n=2$ yields the oscillation frequency:

$\omega_{2}=\sqrt{\frac{8 \sigma}{\rho_{l} R_{0}^{3}}}$

It should be noticed that the linear theory predicts for the mode $n=2$ a shape that is only approximately an oblate or prolate spheroid, although the difference for small oscillation amplitude (i.e. for the range where the linear theory holds) is almost negligible. Moreover, non linear theory [23] yields more accurate (but more complex) results, and among others the fact that the time spent by a drop in the oblate shape is larger than the time spent in a prolate one.

Remaining in the framework of linear theory, the drop shape variation of an axis-symmetric oscillating drop can be expressed through a perturbation parameter $a_{n}(t)=a_{n}^{0} e^{i \omega_{n} t}$ by the equation [39]:

$R(\theta)=R_{0}\left(1+\sum_{n=2} \frac{a_{n}(t)}{2 n+1} P_{n}(\cos \theta)\right)$

where $P_{n}(x)$ are the Legendre polynomials.

The ratio between the surface of the spheroid and that of the isovolumic spherical drop can be calculated as function of time by the following approximation:

$\beta(t)=\frac{A_{\text {spheroid }}}{A_{\text {sphere }}}=1+\frac{2}{5}\left(a_{2}^{0}\right)^{2} \sin ^{2}\left(\omega_{2} t\right)=1+\Delta \beta \sin ^{2}\left(\omega_{2} t\right)$

where $\Delta \beta$ is the non-dimensional maximum excess area from the spherical state.

Referring to Eqs. (3) and (9), the factor $\Gamma$ in Eqs. (4) and (5) can be considered a function of $\beta$.

Under the assumption of quasi-steady drop evaporation, the nondimensional instantaneous evaporation rate depends on time by the relation:

$\hat{m}_{e v}=\Gamma(\beta(t)) \ln \left(\frac{1-\chi_{v, \infty}}{1-\chi_{v, s}}\right)=\Gamma(\beta(t)) \hat{m}_{e v}^{\text {sphere }}$ where $\beta(t)$ is given by Eq. (11). The analogous relation for the nondimensional sensible heat rate can be written:

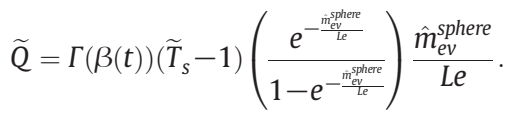

Fig. 3 reports an example of time variation of evaporation rate, in terms of the above defined parameter $\Theta$, over an oscillation period $\left(\tau_{\text {oscill }}\right)$. The drop oscillates between a prolate shape and an oblate shape. As above-mentioned, non-linear analysis found that drops, which are released from an initially two lobed configuration, spend less time in prolate form than in oblate one [23]. However, in the present investigation it will be assumed, following the results of linear theory, that the time spent in prolate state is equal to the time spent in oblate state. In Fig. 3 the maximum value of the parameter $\beta$ during the oscillation period is fixed equal to 1.1 , which corresponds to a maximum value of $\varepsilon$ equal to $\sim 2.2$ in the prolate state and a minimum value equal to $\sim 0.49$ in the oblate one. The results enlighten that, for the chosen values of $\Delta \beta$, the maximum evaporation rate exceeds that of an iso-volumic spherical drop of about $6 \%$ and $4 \%$ when the drop is in the prolate and oblate states, respectively.

Mashayek [34] proposed a correlation for the instantaneous nondimensional evaporation rate of oscillating drop, derived from numerical simulations:

$\hat{m}_{e v}^{\text {Mashayek }[34]}=\left[G_{n} \epsilon_{n}^{2}(1+\cos (2 \omega t))+1\right] \ln \left(\frac{1-\chi_{v, \infty}}{1-\chi_{v, s}}\right)$

where $G_{n}$ is function of the oscillation mode and it is found to be equal to 0.6 for mode $n=2$ [34], while the surface disturbance amplitude is calculated as a function of the drop surface ratio $\beta$ :

$\epsilon_{n}=\sqrt{\frac{\beta-1}{0.8}}$.

The ratio between the instantaneous non-dimensional evaporation rate of oscillating drop calculated by the correlation of Mashayek [34] (Eq. (14)) and the corresponding one from an iso-volumic spherical drop is also plotted in Fig. 3, over an oscillation period. The graph enlightens that this approximated model does not take into account the different evaporation rates from oblate and prolate drops and, except when the drop approaches the spherical shape, it always overestimates the results, with a maximum deviation from the present model of about $10 \%$.

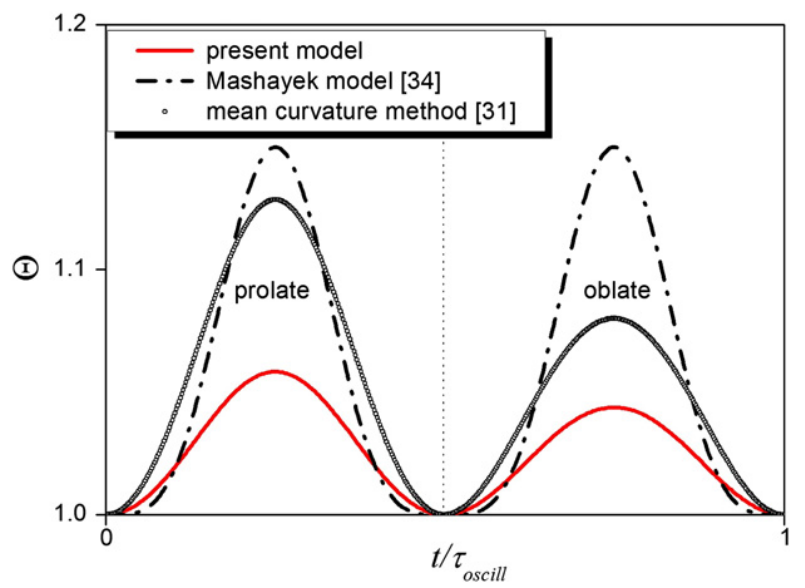

Fig. 3. Instantaneous non-dimensional evaporation rate, during a drop oscillation period, predicted by the present model, the correlation of Mashayek [34] and the mean curvature method [31]. $\beta_{\max }=1.1$. 
Fig. 3 also shows the results obtained assuming that the drop evaporation rate can be calculated using the mean curvature model of Mashayek [31], integrating Eq. (6) along the drop surface:

$\hat{m}_{e v}^{\text {Mashayek }[31]}=\frac{\varepsilon^{2 / 3}}{4} \Upsilon \hat{m}_{e v}^{\text {sphere }}$

where:

$$
\begin{aligned}
\Upsilon^{\text {oblate }} & =2+\frac{2}{\varepsilon \sqrt{1-\varepsilon^{2}}} \arctan \frac{\sqrt{1-\varepsilon^{2}}}{\varepsilon} \\
\Upsilon^{\text {prolate }} & =2+\frac{1}{\varepsilon \sqrt{\varepsilon^{2}-1}} \ln \frac{\varepsilon+\sqrt{\varepsilon^{2}-1}}{\varepsilon-\sqrt{\varepsilon^{2}-1}} .
\end{aligned}
$$

The mean curvature model clearly distinguishes between the two drop shapes and it overestimates, respect to the exact solution, the evaporation rate along the whole oscillation period, with a maximum deviation of about $7 \%$ during the prolate state and about $4 \%$ during the oblate one.

Finally Fig. 4 shows the average evaporation rate, along one oscillation period, in terms of the parameter $\Theta$ as a function of $\Delta \beta$, as predicted by the present model, which is based on the instantaneous vapour flux calculation from the exact solution of Eq. (1) for spheroidal drops, and by the two approximate models from Mashayek, $[31,34]$. The excess area $\Delta \beta$ has been increased up to $100 \%$, which corresponds to a maximum and a minimum value of $\varepsilon$ equal to about 16.2 and 0.14 , respectively.

The results show that the present model estimates a monotonic in crement of the average evaporation rate, where the maximum excess surface $(\Delta \beta)$ increases. The maximum increment of the evaporation rate is up to about $30 \%$ for $\Delta \beta$ equal to $100 \%$.

The approximate model of Mashayek [34] and the mean curvature model, based on the assumption that the evaporation mass flux is proportional to the mean drop curvature [31], are in close agreement up to an excess area of about $50 \%$, above which the mean curvature model significantly overestimates the evaporation rate.

Both models $[31,34]$ predict higher increment of average evaporation rate due to drop oscillation, with a rising deviation from the present model as $\Delta \beta$ increases, up to $20 \%$ (with the approximate model [34]) and $30 \%$ (with the curvature method [31]) for $\Delta \beta$ equal to $100 \%$. To notice that an analogous curve can be obtained for the average sensible heat rate, along one oscillation period, as function of $\Delta \beta$.

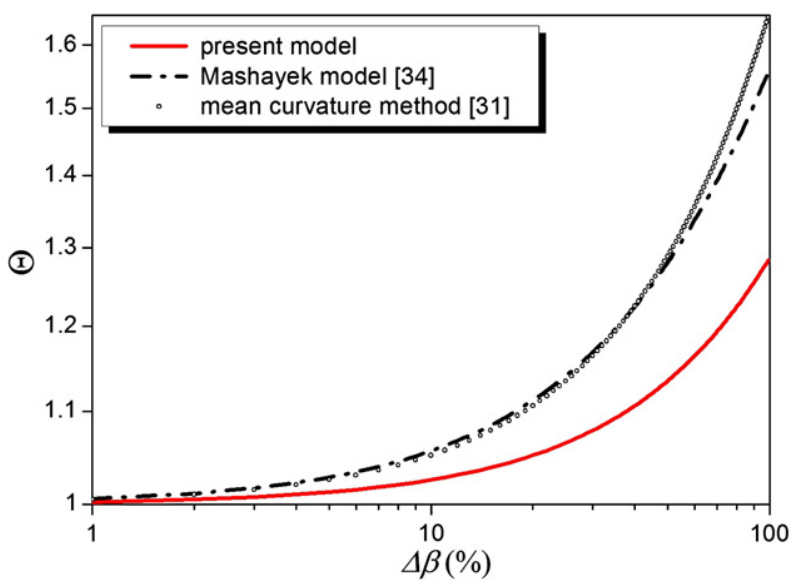

Fig. 4. Average non-dimensional evaporation rate, along an oscillation period, as a function of the excess area $\Delta \beta$, predicted by the present model, the correlation of Mashayek [34] and the mean curvature method [31].

\section{The applicability of the quasi-steady state assumption}

All evaporation models for liquid drop oscillating in a gaseous environment assume that the process occurs under quasi-steady state conditions. To the best of authors' knowledge the only numerical model relaxing this hypothesis is proposed in [12], and no investigations are available in the open literature discussing such approximation.

In the present investigation the estimation of the instantaneous vapour flux from an oscillating drop by Eq. (2) is based on a quasisteady-state assumption. Such hypothesis may hold when the characteristic time scale of the oscillation process is much larger than that of the evaporation one.

The characteristic time scale of the oscillatory process (assuming $n=2$ ) can be defined as the inverse of the oscillation frequency:

$\tau_{\text {oscill }} \approx \sqrt{\frac{\rho_{l} R_{0}^{3}}{\sigma}}$

and this should be compared to the evaporation time scale to check the validity of the quasi-steady-state assumption, that may be acceptable when:

$t_{\text {evap }} \ll t_{\text {oscill }}$.

Evaporation is an overlapping of mass and thermal diffusive phenomena (driven by the coefficients $D_{v}$ and $\alpha$, respectively) and a convective one (i.e. the Stefan flow, driven by a characteristic velocity $U_{0}=\frac{\dot{m}_{e v}}{\beta 4 \pi R_{0}^{2} \rho}=\frac{\hat{m}_{e v} D_{v}}{R_{0}}$. From these two parameters and the drop size, three different (but not independent) characteristic times can be defined for both mass and heat transfer processes:

$\begin{array}{ccc}\begin{array}{cll}\text { Characteristictimes } \\ \text { convective }\end{array} & \text { Mass transfer } & \text { Heat transfer } \\ \text { diffusive } & t_{\text {conv }} \approx \frac{R_{0}}{U} & t_{\text {conv }}^{H} \approx \frac{R_{0}}{U} \\ \text { convective-diffusive } & t_{\text {diff }} \approx \frac{R_{0}^{2}}{D_{v}} & t_{\text {diff }}^{H} \approx \frac{R_{0}^{2}}{\alpha}=\frac{t_{\text {diff }}}{\text { Le }} \\ t_{\mathrm{cd}} \approx \frac{D_{v}}{U^{2}}=\frac{t_{\text {conv }}^{2}}{t_{\text {diff }}} & t_{\mathrm{cd}}^{H} \approx \frac{\alpha}{U^{2}}=t_{\mathrm{cd}} \text { Le }\end{array}$

and a re-arrangement yields:

Mass transfer

$t_{\text {conv }} \approx \frac{\beta R_{0}^{2}}{\hat{m}_{e v} D_{v}}=\frac{\beta t_{\text {diff }}}{\hat{m}_{e v}}$

$t_{c d} \approx \frac{\beta^{2} R_{0}^{2}}{\hat{m}_{e v}^{2} D_{v}}=\frac{\beta^{2} t_{d i f f}}{\hat{m}_{e v}^{2}}$

and since $\frac{\beta}{\hat{m}_{e v}}>1$ (for $\varepsilon \lesssim 42.7$, that stems from Eq. (4)), then:

$\left(\frac{\beta}{\Gamma(\beta)}\right)^{2} \frac{R_{0}^{2}}{\left(\hat{m}_{e v}^{\text {sphere }}\right)^{2} D_{v}} \max [1, L e]=\max \left[t_{c d}, t_{c d}^{H}\right]>\max \left[t_{\text {conv }}, t_{\text {conv }}^{H}\right]>\max \left[t_{\text {diff }}, t_{\text {diff }}^{H}\right]$.

The evaporation characteristic time scale $t_{\text {evap }}$ may be one of the three above-mentioned characteristic times, but the condition (16) is certainly satisfied if $\max \left[t_{c d}, t_{c d}^{H}\right] \ll t_{\text {oscill. }}$.

To quantify the applicability of the quasi-steady state assumption, consider the case with $\chi_{v, \infty}=0$, and $P_{T, s}=P_{T, \infty}$. Since:

$\chi_{v, s}=\frac{\rho_{v, s}}{\rho_{s}}=\frac{P_{v, s} M m_{v}}{P_{v, s} M m_{v}+\left(P_{T, \infty}-P_{v, s}\right) M m_{g}}$ 
and after observing that $1<\frac{\beta}{\Gamma(\beta)}<1.2$ (for $\varepsilon \gtrsim 0.2$, which is a reasonable value for the maximum drop deformation in realistic application test cases), the limiting condition is:

$\frac{D_{v}^{2}\left(T_{f i m}\right) \rho_{l} \ln ^{4}\left(1-\chi_{v, s}\right)}{2.074 \sigma\left(T_{s}\right)}=R_{\text {lim }} \ll R_{0}$

where $T_{\text {film }}=\frac{2 T_{s}+T_{\infty}}{3}$, according to the " $1 / 3$ rd rule" suggested by [40]. The mass diffusivity in the gas phase is a function of the temperature, with an empirical correlation proposed by [41] $D_{v}\left(T_{f i l m}\right)=D_{v, 0}\left(\frac{T_{f i m}}{T_{0}}\right)^{m}$, where $m=\frac{3}{2}$ then:

$T_{\infty}=3 T_{0}\left(\frac{1.2^{4} \sigma\left(T_{s}\right) R_{\text {lim }} \max \left[1, L e^{2}\right]}{D_{v, 0}^{2} \rho_{l} \ln ^{4}\left(1-\chi_{v, s}\right)}\right)^{\frac{1}{2 m}}-2 T_{s}$

yielding a relation between the gas temperatures at free stream conditions, $T_{\infty}$, and at drop surface, $T_{s}$, for each value of the drop radius $R_{\mathrm{lim}}$, allowing to define a region on a $T_{\infty}-T_{s}$ graph where the assumption (16) may be accepted.
The graphs of Fig. 5 report for different drop species (water, methanol, ethanol, acetone, benzene, hexane, octane, dodecane and hexadecane) the range of drop and gas temperatures, relative to each drop size, where the above discussed condition may hold: for any curve relative to a given drop size, the gas temperature must be larger than the reported limit, as a function of the drop temperature.

An inspection on the values of such characteristic times shows that the inequality may acceptably hold for small drops (few tenths of micron) in hot gaseous environment and for high volatility fluids.

These results show that the proposed model offers a rather easy-toimplement analytical expression for the instantaneous and average evaporation rate and vapour flux from oblate and prolate oscillating spheroidal liquid drops, making it suitable for more complex spray simulations.

\section{Conclusions}

The evaporation of spheroidal drop in gaseous atmosphere has been investigated, calculating the exact solution for the instantaneous vapour flux and sensible heat rate under steady-state conditions as function of the increment of surface area respect to iso-volumic spherical drops.

The effect of drop oscillation between oblate and prolate states on evaporation was taken into account deriving a model based on the
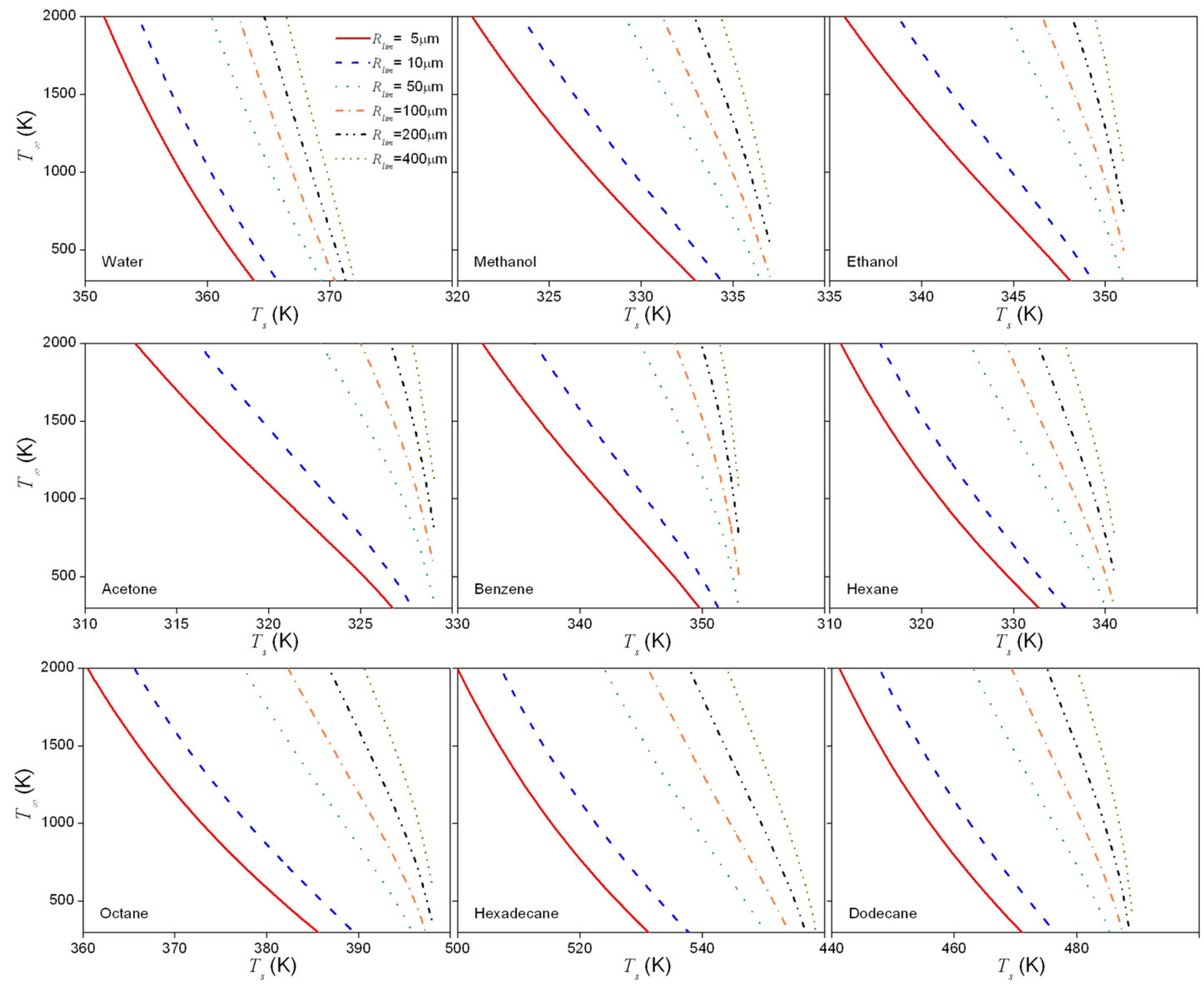

Fig. 5. Maps, for different liquids, of gas and drop temperature and drop size for the validity of the quasi-steady-state assumption. 
linear theory and assuming quasi steady-state drop evaporation. The model calculates the instantaneous and average, over an oscillation period, mass and heat rates, function of the oscillating frequency and drop deformation. The results have been compared with the predictions from approximate models available in the literature.

The present model, which is able to capture the different evaporating mechanisms from oblate and prolate drops, estimates a monotonic increment of the average evaporation rate as the maximum excess surface area increases. Moreover it predicts an increment of average evaporation rate and sensible heat rate due to drop oscillation, up to $20 \%$ for a maximum excess surface area equal to $100 \%$.

The quasi steady-state assumption is finally discussed calculating for different fluids the range of drop size and temperature, and gas temperature where the assumption may be acceptable. The results enlighten that it is valid for small, high volatility liquid drops, evaporating under high gas temperature conditions, typical of spray combustion applications.

\section{Acknowledgements}

This work was partially financed by the Project V.I.R. Berg. cofinanced by Regione Lombardia.

\section{References}

[1] C.T. Crowe, M. Sommerfeld, Y. Tsuji, Multiphase Flows With Droplets and Particles, CRC Press, Boca Raton, 1998

[2] J.C. Maxwell, Diffusion, Encyclopaedia Britannica, ninth ed., vol. 7, 1877, p. 214.

[3] N.A. Fuchs, Vaporisation and Droplet Growth in Gaseous Media, Pergamon Press, London, 1959.

[4] W.A. Sirignano, Fluid Dynamics and Transport of Droplets and Sprays, Cambridge University Press, 1999

[5] A.Y. Tong, W.A. Sirignano, Multicomponent transient droplet vaporization with internal circulation: integral equation formulation, Numer, Heat Tran. 10 (1986) 253-278.

[6] Y.Zeng, C.F. Lee, Multicomponent-fuel film-vaporization model for multidimensional computations, J. Propuls. Power 16 (2000) 964-973.

[7] B. Abramzon, W.A. Sirignano, Droplet vaporization model for spray combustion calculations, Int. J. Heat Mass Transfer 32 (9) (1989) 1605-1618.

[8] G. Zhu, S.K. Aggarwal, Transient supercritical droplet evaporation with emphasis on the effects of equation of state, Int. J. Heat Mass Transfer 43 (2000) 1157-1171.

[9] S.K. Aggarwal, H.C. Mongia, Multicomponent and high-pressure effects on droplet vaporization, J. Eng. Gas Turbines Power 24 (2002) 248-255.

[10] S.S. Sazhin, Advanced models of fuel droplet heating and evaporation, Prog. Energy Combust. Sci. 32 (2006) 162-214.

[11] M. Hase, B. Weigand, Transient heat transfer of deforming droplets at high Reynolds numbers, Int. J. Numer. Methods Heat Fluid Flow 14 (1) (2004) 85-97.

[12] J. Schlottke, B. Weigand, Direct numerical simulation of evaporating droplets, J. Comput. Phys. 227 (2008) 5215-5237.

[13] S.M. Jeng Z Z Deng Numerical simulation of deformed droplet dynamics and evaporation, Recent Advances in Spray Combustion: Spray Combustion Measurements and Model Simulation, 21996. 305-328.
[14] M.E. Fraser, W.K. Lu, A.E. Hamielec, R. Murarka, Surface tension measurement on pure liquid iron and nickel by an oscillating drop technique, Metall. Trans. 2 (1971) 817-823.

[15] H. Lamb, Hydrodynamics, 6th ed. Cambridge University Press, 1932.

[16] A. Prosperetti, Free oscillations of drops and bubbles: the initial-value problem, J. Fluid Mech. 100 (1980) 333-347.

[17] J.A. Tsamopoulos, R.A. Brown, Nonlinear oscillations of inviscid drops and bubbles, J. Fluid Mech. 127 (1983) 519-537.

[18] T.S. Lundgren, N.N. Mansour, Oscillations of drops in zero gravity with weak viscous effects, J. Fluid Mech. 194 (1991) 479-510.

[19] T.G. Wang, A.V. Anilkumar, C.P. Lee, Oscillations of liquid drops: results from USML-1 experiments in space, J. Fluid Mech. 308 (1996) 1-14.

[20] F. Mashayek, N. Ashgriz, Nonlinear oscillations of drops with internal circulation, Phys. Fluids 10 (5) (1998) 1071-1082.

[21] E.H. Trinh, D.B. Thiessen, R.G. Holt, Driven and freely decaying nonlinear shape oscillations of drops and bubbles immersed in a liquid: experimental results, J. Fluid Mech. 364 (1998) 253-272.

[22] A. Prosperetti, Linear oscillations of constrained drops, bubbles, and plane liquid surfaces, Phys. Fluids 24 (3) (2012)

[23] O. Basaran, Nonlinear oscillations of viscous liquid drops, J. Fluid Mech. 241 (1992) 169-198.

[24] M. Dai, J.B. Perot, D.P. Schmidt, Heat transfer within deforming droplets, Proceedings of ASME: Internal Combustion Engine Division, New Orleans, September, 2002.

[25] G. Guan, J. Zhu, S. Xia, Z. Feng, E.J. Davis, Simulation of mass transfer from an oscillating microdroplet, Int. J. Heat Mass Transfer 48 (2005) 1705-1715.

[26] U. Olgac, D. Izbassarov, M. Muradoglu, Direct numerical simulation of an oscillating droplet in partial contact with a substrate, Comput. Fluids 77 (2013) 152-158.

[27] R. Takaki, N. Yoshiyasu, Y. Arai, K. Adachi, Dynamic pattern formation of an evaporating drop, Proceedings of the First International Symposium for Science on Form, KTK Scientific Publishers, Tokyo, 1986.

[28] R.J. Haywood, M. Renksizbulut, G.D. Raithby, Transient deformation and evaporation of droplets at intermediate Reynolds numbers, Int. J. Heat Mass Transfer 37 (9) (1994) 1401-1409.

[29] T.A. Kowalewski, Transient evaporation of oscillating droplet, Advanced Modelling and Simulation in Engineering, Pultusk1994.

[30] Z.T. Deng, R.J. Litchford, S.M. Jeng, Two-dimensional simulation of droplet evaporation at high pressure, AIAA Paper 29-3122, 1992.

31] F. Mashayek, Dynamics of evaporating drops. Part I: formulation and evaporation model, Int. J. Heat Mass Transfer 44 (8) (2001) 1517-1526.

[32] Z.W. Lian, R.D. Reitz, The effect of vaporization and gas compressibility on liquid jet atomization, Atomization Sprays 3 (3) (1993) 249-264.

[33] S. Tonini, G.E. Cossali, An exact solution of the mass transport equations for spheroida evaporating drops, Int. J. Heat Mass Transfer 60 (2013) 236-240.

[34] F. Mashayek, Dynamics of evaporating drops. Part II: free oscillations, Int. J. Heat Mass Transfer 44 (8) (2001) 1527-1541.

[35] J.C. Slattery, Momentum, Energy and Mass Transfer in Continua, second ed.vol. 482 R. Krieger Publ., New York, 1981.

[36] W.A. Sirignano, Fluid Dynamics and Transport of Droplets and Sprays, second ed. Cambridge University Press, 2010.

[37] S. Tonini, G.E. Cossali, An analytical model of liquid drop evaporation in gaseous environment, Int. J. Therm. Sci. 57 (2012) 45-53.

[38] H. Guggenheimer, Differential Geometry1977. Dover Publications.

[39] E. Becker, W.J. Hiller, T.A. Kowalewski, Nonlinear dynamics of viscous droplets, J. Fluid Mech. 258 (1994) 191-216.

[40] M.C. Yuen, L.W. Chen, On drag of evaporating droplets, Combust. Sci. Technol. 14 (1976) $147-154$.

[41] S. Chapman, T.G. Cowling. The Mathematical Theory of Non-uniform Gases: An Account of the Kinetic Theory of Viscosity, Thermal Conduction, and Diffusion in Gases, Cambridge University Press, 1990. 